\title{
Reconstruction of the Volterra-type integro-differential operator from nodal points
}

\section{Baki Keskin * (D)}

\section{"Correspondence:}

bkeskin@cumhuriyet.edu.tr

Department of Mathematics,

Faculty of Science, Cumhuriyet

University, Sivas, Turkey

\begin{abstract}
In this work, the Sturm-Liouville problem perturbated by a Volterra-type integro-differential operator is studied. We give a uniqueness theorem and an algorithm to reconstruct the potential of the problem from nodal points (zeros of eigenfunctions).
\end{abstract}

MSC: 34A55; 34B07; 34B24; 34B37; 34K29; 34K10; 47G20

Keywords: Sturm-Liouville equation; Inverse nodal problem; Integro-differential equation

\section{Introduction}

We consider the boundary value problem $L$ generated by the convolution-type SturmLiouville integro-differential operator

$$
-y^{\prime \prime}+q(x) y+\int_{0}^{x} M(x, t) y^{\prime}(t) d t=\lambda y, \quad x \in(0, \pi)
$$

with boundary conditions

$$
\begin{aligned}
& y^{\prime}(0)-h y(0)=0, \\
& y^{\prime}(\pi)+H y(\pi)=0
\end{aligned}
$$

and with the discontinuity conditions

$$
\left\{\begin{array}{l}
y\left(\frac{\pi}{2}+0\right)=\alpha y\left(\frac{\pi}{2}-0\right), \\
y^{\prime}\left(\frac{\pi}{2}+0\right)=\alpha^{-1} y^{\prime}\left(\frac{\pi}{2}-0\right),
\end{array}\right.
$$

where $\lambda$ is the spectral parameter; $\alpha$ is a positive real constant; $q(x)$ and $M(x, t)$ are realvalued functions from the class $L_{2}(0, \pi)$ and $W_{2}^{1}(0, \pi)$, respectively. Without loss of generality, we assume that $\int_{0}^{\pi}(q(x)+M(x, x)) d x=0$.

The first result of the inverse nodal Sturm-Liouville problem was given by McLaughlin in [1]. In this work, she proved that the potential of the considered problem can be uniquely determined by a given dense subset of the zeros of the eigenfunctions called

(c) The Author(s) 2018. This article is distributed under the terms of the Creative Commons Attribution 4.0 International License (http://creativecommons.org/licenses/by/4.0/), which permits unrestricted use, distribution, and reproduction in any medium, provided you give appropriate credit to the original author(s) and the source, provide a link to the Creative Commons license, and indicate if changes were made. 
nodal points. In 1989, Hald and McLaughlin studied more general boundary conditions and gave some numerical schemes for the reconstruction of the potential from a given dense subset of nodal points [2]. Yang provided an algorithm to determine the coefficients of the Sturm-Liouville problem by using the given nodal points in [3]. Inverse nodal problems for different types of operators have been extensively well studied in several papers (see [4-14] and [15]).

Inverse problems for integro-differential operators and the other classes of nonlocal operators are more difficult to investigate. The classical methods are often not applicable for such problems. In present, the studies concerning the perturbation of a differential operator by a Volterra-type integral operator, namely the integro-differential operator, continue to be performed and are beginning to have a significant place in the literature (see [1621], and [22]). Inverse nodal problem for this type of operator was first discussed by [23]. It is shown in this study that the potential function can be determined by using nodal points, while the coefficient of the integral operator is known. The inverse nodal problem for Dirac-type integro-differential operators was first investigated by [24]. In this work, it is shown that the coefficients of the differential part of the operator can be determined by using nodal points, and nodal points also give partial information about the integral part.

In the present paper we investigate the inverse nodal problem for Volterra-type integrodifferential operator. This type of operator has previously been addressed in [25] and [26].

\section{Preliminaries}

Let $\varphi(x, \lambda)$ be the solution of (1) with the initial conditions

$$
\varphi(0, \lambda)=1, \quad \varphi^{\prime}(0, \lambda)=h
$$

and the jump conditions (4).

We have the following integral equations of the solution of (1): for $x<\frac{\pi}{2}$,

$$
\begin{aligned}
\varphi(x, \lambda)= & \cos \sqrt{\lambda} x+h \frac{\sin \sqrt{\lambda} x}{\sqrt{\lambda}}+\int_{0}^{x} \frac{\sin \sqrt{\lambda}(x-t)}{\sqrt{\lambda}} q(t) \varphi(t, \lambda) d t \\
& +\int_{0}^{x} \frac{\sin \sqrt{\lambda}(x-t)}{\sqrt{\lambda}} \int_{0}^{t} M(t, \tau) \varphi^{\prime}(\tau, \lambda) d \tau d t
\end{aligned}
$$

for $x>\frac{\pi}{2}$,

$$
\begin{aligned}
\varphi(x, \lambda)= & \alpha^{+} \cos \sqrt{\lambda} x+\alpha^{-} \cos \sqrt{\lambda}(\pi-x) \\
& +\frac{h}{\sqrt{\lambda}}\left[\alpha^{+} \sin \sqrt{\lambda} x+\sin \sqrt{\lambda}(\pi-x)\right] \\
& +\int_{0}^{\pi / 2}\left[\alpha^{+} \frac{\sin \sqrt{\lambda}(x-t)}{\sqrt{\lambda}}+\alpha^{-} \frac{\sin \sqrt{\lambda}(\pi-x-t)}{2 \sqrt{\lambda}}\right] q(t) \varphi(t, \lambda) d t \\
& +\int_{0}^{\pi / 2} \int_{0}^{t}\left[\alpha^{+} \frac{\sin \sqrt{\lambda}(x-t)}{\sqrt{\lambda}}+\alpha^{-} \frac{\sin \sqrt{\lambda}(\pi-x-t)}{2 \sqrt{\lambda}}\right] M(t, \tau) \varphi^{\prime}(\tau, \lambda) d \tau d t \\
& +\int_{\pi / 2}^{x}+\frac{\sin \sqrt{\lambda}(x-t)}{\sqrt{\lambda}} q(t) \varphi(t, \lambda) d t \\
& +\int_{\pi / 2}^{x} \int_{0}^{t} \frac{\sin \sqrt{\lambda}(x-t)}{\sqrt{\lambda}} M(t, \tau) \varphi^{\prime}(\tau, \lambda) d \tau d t,
\end{aligned}
$$


where $\alpha^{ \pm}=\frac{1}{2}\left(\alpha \pm \frac{1}{\alpha}\right)$. By virtue of the above equations, we have the following asymptotic relations for sufficiently large $|\lambda|$ : for $x<\frac{\pi}{2}$,

$$
\begin{aligned}
\varphi(x, \lambda)= & \cos \sqrt{\lambda} x+\frac{\sin \sqrt{\lambda} x}{2 \sqrt{\lambda}}\left(2 h+\int_{0}^{x} q(t) d t+\int_{0}^{x} M(t, t) d t\right) \\
& +o\left(\frac{1}{\sqrt{\lambda}} \exp |\tau| x\right) ;
\end{aligned}
$$

for $x>\frac{\pi}{2}$,

$$
\begin{aligned}
\varphi(x, \lambda)= & \alpha^{+} \cos \sqrt{\lambda} x+\alpha^{-} \cos \sqrt{\lambda}(\pi-x) \\
& +\alpha^{+} \frac{\sin \sqrt{\lambda} x}{2 \sqrt{\lambda}} I_{1}(x)+\alpha^{-} I_{2}(x)+o\left(\frac{1}{\sqrt{\lambda}} \exp |\tau| x\right),
\end{aligned}
$$

where $I_{1}(x)=2 h+\int_{0}^{x} q(t) d t+\int_{0}^{x} M(t, t) d t, I_{2}(x)=\int_{0}^{\pi / 2}(q(t)+M(t, t)) d t-\int_{\pi / 2}^{x}(q(t)+$ $M(t, t)) d t$, and $\tau=|\operatorname{Im} \sqrt{\lambda}|$.

Define a function $\Delta(\lambda)$ as follows:

$$
\Delta(\lambda):=\varphi^{\prime}(\pi, \lambda)+H \varphi(\pi, \lambda)
$$

This entire function is called a characteristic function of the problem $L$ and the zeros of it are eigenvalues of the problem $L$. For sufficiently large $|\lambda|$, by virtue of (8) and (9), we have the following asymptotic formula:

$$
\Delta(\lambda)=-\alpha^{+}\left\{\sqrt{\lambda} \sin \sqrt{\lambda} \pi-\frac{\delta_{1}}{2} \cos \sqrt{\lambda} \pi+\frac{\delta_{2}}{2}\right\}+o(\exp |\tau| \pi)
$$

where

$$
\begin{aligned}
& \delta_{1}=2 h+\alpha^{+} H+\int_{0}^{\pi}(q(t)+M(t, t)) d t, \\
& \delta_{2}=\frac{\alpha^{-}}{\alpha^{+}}\left[-2 H+\int_{0}^{\pi}(q(t)+M(t, t)) d t-2 \int_{0}^{\pi / 2}(q(t)+M(t, t)) d t\right] .
\end{aligned}
$$

It can be easily shown that the sequence $\left\{\lambda_{n}\right\}_{n \geq 0}$ satisfies the following asymptotic relation for $n \rightarrow \infty$ :

$$
\sqrt{\lambda_{n}}=n+\frac{\mu_{n}}{2 n \pi}+o\left(\frac{1}{n}\right)
$$

and

$$
\frac{1}{\sqrt{\lambda_{n}}}=\frac{1}{n}-\frac{\mu_{n}}{2 n^{3} \pi}+o\left(\frac{1}{n^{3}}\right)
$$

where $\mu_{n}=\delta_{1}+(-1)^{n} \delta_{2}$.

Lemma 1 The eigenfunction $\varphi\left(x, \lambda_{n}\right)$ corresponding to the eigenvalue $\lambda_{n}$ has exactly $n$ zeros $\left\{x_{n}^{j}: n \geq 1, j=\overline{0, n-1}\right\}$, namely nodal points, in $(0, \pi)$, such that $0<x_{n}^{0}<x_{n}^{1}<\cdots<x_{n}^{n-1}<\pi$ 
and the numbers $\left\{x_{n}^{j}\right\}$ have the following asymptotic formulae for sufficiently large $n$ : for $x_{n}^{j} \in\left(0, \frac{\pi}{2}\right)$,

$$
x_{n}^{j}= \begin{cases}\frac{(j+1 / 2) \pi}{n}-\frac{(j+1 / 2) \pi}{n} \frac{\left(\delta_{1}+\delta_{2}\right)}{2 n^{2} \pi}+\frac{I_{1}\left(x_{n}^{j}\right)}{2 n^{2}}+o\left(\frac{1}{n^{2}}\right), & n=2 k, k \in \mathbb{Z}, \\ \frac{(j+1 / 2) \pi}{n}-\frac{(j+1 / 2) \pi}{n} \frac{\left(\delta_{1}-\delta_{2}\right)}{2 n^{2} \pi}+\frac{I_{1}\left(x_{n}^{j}\right)}{2 n^{2}}+o\left(\frac{1}{n^{2}}\right), & n=2 k+1, k \in \mathbb{Z} ;\end{cases}
$$

and for $x_{n}^{j} \in\left(\frac{\pi}{2}, \pi\right)$,

$$
x_{n}^{j}= \begin{cases}\frac{(j+1 / 2) \pi}{n}-\frac{(j+1 / 2) \pi}{n} \frac{\left(\delta_{1}+\delta_{2}\right)}{2 n^{2} \pi}+\frac{\alpha^{-}\left(\delta_{1}+\delta_{2}\right)+\alpha^{+} I_{1}\left(x_{n}^{j}\right)-\alpha^{-} I_{2}\left(x_{n}^{j}\right)}{2 \rho_{n} n^{2}}+o\left(\frac{1}{n^{2}}\right), & n=2 k, k \in \mathbb{Z}, \\ \frac{(j+1 / 2) \pi}{n}-\frac{(j+1 / 2) \pi}{n} \frac{\left(\delta_{1}-\delta_{2}\right)}{2 n^{2} \pi}+\frac{-\alpha^{-}\left(\delta_{1}-\delta_{2}\right)+\alpha^{+} I_{1}\left(x_{n}^{j}\right)+\alpha^{-} I_{2}\left(x_{n}^{j}\right)}{2 \rho_{n} n^{2}}+o\left(\frac{1}{n^{2}}\right), & n=2 k+1, k \in \mathbb{Z},\end{cases}
$$

where $\rho_{n}=\alpha^{+}+(-1)^{n} \alpha^{-}$.

Proof By virtue of (8) and (9), we get the following asymptotic formula for eigenfunction $\varphi\left(x, \lambda_{n}\right)$ :

$$
\begin{aligned}
\varphi\left(x, \lambda_{n}\right)= & \cos \sqrt{\lambda_{n}} x+\frac{\sin \sqrt{\lambda_{n}} x}{\sqrt{\lambda_{n}}} I_{1}(x)+o\left(\frac{e^{|\tau| x}}{\sqrt{\lambda_{n}}}\right) \quad \text { for } x<\frac{\pi}{2}, \\
\varphi\left(x, \lambda_{n}\right)= & \alpha^{+} \cos \sqrt{\lambda_{n}} x+\alpha^{-} \cos \sqrt{\lambda_{n}}(\pi-x)+\frac{\alpha^{+} \sin \sqrt{\lambda_{n}} x}{2 \sqrt{\lambda_{n}}} I_{1}(x) \\
& +\frac{\alpha^{-} \sin \sqrt{\lambda_{n}}(\pi-x)}{2 \sqrt{\lambda_{n}}} I_{2}(x)+o\left(\frac{e^{|\tau| x}}{\sqrt{\lambda_{n}}}\right) \quad \text { for } x<\frac{\pi}{2}
\end{aligned}
$$

for sufficiently large $n$, uniformly in $x$. Since the zeros of eigenfunctions are nodal points, from $\varphi\left(x_{n}^{j}, \lambda_{n}\right)=0$, we get

$$
\begin{aligned}
& \alpha^{+} \cos \sqrt{\lambda_{n}} x_{n}^{j}+\alpha^{-} \cos \sqrt{\lambda_{n}}\left(\pi-x_{n}^{j}\right)+\frac{\alpha^{+} \sin \sqrt{\lambda_{n}} x_{n}^{j}}{\sqrt{\lambda_{n}}} I_{1}\left(x_{n}^{j}\right) \\
& +\frac{\alpha^{-} \sin \sqrt{\lambda_{n}} \pi \cos \sqrt{\lambda_{n}} x_{n}^{j}}{2 \sqrt{\lambda_{n}}} I_{2}\left(x_{n}^{j}\right)-\frac{\alpha^{-} \sin \sqrt{\lambda_{n}} x_{n}^{j} \cos \sqrt{\lambda_{n}} \pi}{2 \sqrt{\lambda_{n}}} I_{2}\left(x_{n}^{j}\right)+o\left(\frac{e^{|\tau| x_{n}^{j}}}{\sqrt{\lambda_{n}}}\right)=0,
\end{aligned}
$$

which implies that

$$
\begin{gathered}
\cot \sqrt{\lambda_{n}} x_{n}^{j}\left(\alpha^{+}+\alpha^{-} \cos \sqrt{\lambda_{n}} \pi+\frac{\alpha^{-} \sin \sqrt{\lambda_{n}} \pi}{2 \sqrt{\lambda_{n}}} I_{2}\left(x_{n}^{j}\right)\right) \\
=\alpha^{-} \sin \sqrt{\lambda_{n}} \pi-\frac{\alpha^{+}}{2 \sqrt{\lambda_{n}}} I_{1}\left(x_{n}^{j}\right)+\frac{\alpha^{-} \cos \sqrt{\lambda_{n}} \pi}{2 \sqrt{\lambda_{n}}} I_{2}\left(x_{n}^{j}\right)+o\left(\frac{e^{|\tau| x_{n}^{j}}}{\sqrt{\lambda_{n}}}\right), \\
\cot \sqrt{\lambda_{n}} x_{n}^{j}=\frac{-\alpha^{-} \sin \sqrt{\lambda_{n}} \pi-\frac{\alpha^{+}}{2 \sqrt{\lambda_{n}}} I_{1}\left(x_{n}^{j}\right)+\frac{\alpha^{-} \cos \sqrt{\lambda_{n}} \pi}{2 \sqrt{\lambda_{n}}} I_{2}\left(x_{n}^{j}\right)+o\left(\frac{e^{|\tau| x_{n}^{j}}}{\sqrt{\lambda_{n}}}\right)}{\alpha^{+}\left(1+\frac{\alpha^{-}}{\alpha^{+}} \cos \sqrt{\lambda_{n}} \pi+\frac{\alpha^{-} \sin \sqrt{\lambda_{n}} \pi}{2 \alpha^{+} \sqrt{\lambda_{n}}} I_{2}\left(x_{n}^{j}\right)\right)} \\
=\frac{-1}{\rho_{n}}\left(\alpha^{-} \sin \sqrt{\lambda_{n}} \pi+\frac{\alpha^{+}}{2 \sqrt{\lambda_{n}}} I_{1}\left(x_{n}^{j}\right)-\frac{\alpha^{-} \cos \sqrt{\lambda_{n}} \pi}{2 \sqrt{\lambda_{n}}} I_{2}\left(x_{n}^{j}\right)\right)+o\left(\frac{e^{|\tau| x_{n}^{j}}}{\sqrt{\lambda_{n}}}\right),
\end{gathered}
$$


which is equivalent to

$$
\begin{aligned}
\tan \left(\frac{\pi}{2}-\sqrt{\lambda_{n}} x_{n}^{j}\right)= & \frac{-1}{\rho_{n}}\left(\alpha^{-} \sin \sqrt{\lambda_{n}} \pi+\frac{\alpha^{+}}{2 \sqrt{\lambda_{n}}} I_{1}\left(x_{n}^{j}\right)-\frac{\alpha^{-} \cos \sqrt{\lambda_{n}} \pi}{2 \sqrt{\lambda_{n}}} I_{2}\left(x_{n}^{j}\right)\right) \\
& +o\left(\frac{e^{|\tau| x_{n}^{j}}}{\sqrt{\lambda_{n}}}\right)
\end{aligned}
$$

for $x_{n}^{j}>\frac{\pi}{2}$. Taylor's expansions formula for the arctangent yields

$$
\begin{aligned}
\sqrt{\lambda_{n}} x_{n}^{j}= & (j+1 / 2) \pi \\
& +\frac{1}{\rho_{n}}\left(\alpha^{-} \sin \sqrt{\lambda_{n}} \pi+\frac{\alpha^{+}}{2 \sqrt{\lambda_{n}}} I_{1}\left(x_{n}^{j}\right)-\frac{\alpha^{-} \cos \sqrt{\lambda_{n}} \pi}{2 \sqrt{\lambda_{n}}} I_{2}\left(x_{n}^{j}\right)\right) \\
& +o\left(\frac{e^{|\tau| x_{n}^{j}}}{\sqrt{\lambda_{n}}}\right) .
\end{aligned}
$$

If we divide both sides of this equality by $\sqrt{\lambda_{n}}$ and take account of the asymptotic formula of $\sqrt{\lambda_{n}}$, we get

$$
\begin{aligned}
x_{n}^{j}= & \frac{(j+1 / 2) \pi}{n}-\frac{(j+1 / 2) \pi}{n} \frac{\mu_{n}}{2 n^{2} \pi} \\
& +\frac{(-1)^{n} \alpha^{-} \mu_{n}+\alpha^{+} I_{1}\left(x_{n}^{j}\right)-(-1)^{n} \alpha^{-} I_{2}\left(x_{n}^{j}\right)}{2 \rho_{n} n^{2}}+o\left(\frac{1}{n^{2}}\right) .
\end{aligned}
$$

The proof of (14) is completed. Equation (13) can be proved similarly.

Let $\Omega=\Omega_{0} \cup \Omega_{1}$ be the set of zeros of eigenfunction, i.e., $\Omega_{0}=\left\{x_{n}^{j}: n=2 k, k \in \mathbb{Z}\right\}$, $\Omega_{1}=\left\{x_{n}^{j}: n=2 k+1, k \in \mathbb{Z}\right\}$. For each fixed $x \in(0, \pi)$, there exists a sequence $\left(x_{n}^{j(n)}\right) \subset \Omega_{m}$ $(m=0,1)$, which converges to $x$. Therefore, from Lemma 1 , we can show that the following finite limits exist:

$$
\begin{array}{ll}
\lim _{n \rightarrow \infty} 2 n^{2}\left(x_{n}^{j(n)}-\frac{\left(j(n)+\frac{1}{2}\right) \pi}{n}\right)=f_{m}(x) & \text { for } x<\frac{\pi}{2}, \\
\lim _{n \rightarrow \infty} 2 n^{2}\left(x_{n}^{j(n)}-\frac{\left(j(n)+\frac{1}{2}\right) \pi}{n}\right)=g_{m}(x) & \text { for } x>\frac{\pi}{2},
\end{array}
$$

where

$$
\begin{aligned}
& f_{m}(x)=-\frac{\mu_{m} x}{\pi}+I_{1}(x) \quad \text { for } x<\frac{\pi}{2}, \\
& g_{m}(x)=-\frac{\mu_{m} x}{\pi}+I_{1}(x)+\sigma_{m} \quad \text { for } x>\frac{\pi}{2},
\end{aligned}
$$

where $\sigma_{m}=\frac{(-1)^{m} \alpha^{-}\left(\mu_{m}-2 I_{1}\left(\frac{\pi}{2}\right)+2 h\right)}{\rho_{m}}$. Put

$$
F_{m}(x)= \begin{cases}f_{m}(x) & \text { for } x<\frac{\pi}{2}, \\ g_{m}(x) & \text { for } x>\frac{\pi}{2}\end{cases}
$$


The following theorem shows that if one of $q(x)$ or $M(x, x)$ is given, then the other one can be determined uniquely by using a dense subset of the given nodal set.

Theorem 1 The given dense subset of the nodal set $\Omega_{0}\left(\right.$ or $\left.\Omega_{1}\right)$ uniquely determines $q(x)+$ $M(x, x)$, a.e. on $(0, \pi)$ and the coefficients $h, H$, and $\alpha$ of the boundary and discontinuity conditions. $q(x)+M(x, x)$ and the constants $h, H$, and $\alpha$ can be constructed by the following formulae:

1. For each fixed $x \in(0, \pi)$, choose a sequence $\left(x_{n}^{j(n)}\right) \subset \Omega_{0}$, i.e., $\lim _{n \rightarrow \infty} x_{n}^{j(n)}=x$;

2. Find $F_{m}(x)$ from equation (19) and calculate

$$
\begin{aligned}
& h=\frac{F_{0}(0)}{2}, \\
& \mu_{0}=-F_{0}(\pi)+F_{0}(0)-F_{0}\left(\frac{\pi}{2}-0\right)+F_{0}\left(\frac{\pi}{2}+0\right) \\
& q(x)+M(x, x)=F_{0}^{\prime}(x)+\frac{\mu_{0}}{\pi} \\
& \alpha=\sqrt{\frac{F_{0}(0)-2 F_{0}\left(\frac{\pi}{2}-0\right)}{F_{0}(0)-2 F_{0}\left(\frac{\pi}{2}+0\right)}} \\
& 2 I_{1}\left(\frac{\pi}{2}\right)=-F_{0}(\pi)+F_{0}(0)+F_{0}\left(\frac{\pi}{2}-0\right)+F_{0}\left(\frac{\pi}{2}+0\right) \\
& H=\frac{\alpha^{+}\left(\mu_{0}-2 h\right)+2 \alpha^{-}\left(I_{1}(\pi / 2)-F_{0}(0)\right)}{\left(\alpha^{+}\right)^{2}-2 \alpha^{-}} .
\end{aligned}
$$

Example 1 Consider the following BVP:

$$
L:\left\{\begin{array}{l}
-y^{\prime \prime}+q(x) y+\int_{0}^{x} M(x, t) y^{\prime}(t) d t=\lambda y, \quad x \in(0, \pi) \\
y^{\prime}(0)-h y(0)=0 \\
y^{\prime}(\pi)+H y(\pi)=0 \\
y\left(\frac{\pi}{2}+0\right)=\alpha y\left(\frac{\pi}{2}-0\right) \\
y^{\prime}\left(\frac{\pi}{2}+0\right)=\alpha^{-1} y^{\prime}\left(\frac{\pi}{2}-0\right)
\end{array}\right.
$$

where $q(x)$ and $M(x, t)$ are real-valued functions from the class $L_{2}(0, \pi)$ and $W_{2}^{1}(0, \pi)$, respectively, and $h, H, \alpha$ are unknown coefficients we confirmed on the assumptions of the problem $L$. Let $\left\{x_{n}^{j}\right\}$ be the zeros of the eigenfunction of the considered problem in $(0, \pi)$ with the following asymptotics: If $x_{n}^{j} \in\left(0, \frac{\pi}{2}\right)$,

$$
x_{n}^{j}=\frac{(j+1 / 2) \pi}{n}-\frac{(j+1 / 2) \pi}{n} \frac{2}{5 n^{2} \pi}+\frac{2+\sin \left(\frac{j+1 / 2}{n}\right) \pi}{2 n^{2}}+o\left(\frac{1}{n^{2}}\right) .
$$

If $x_{n}^{j} \in\left(\frac{\pi}{2}, \pi\right)$,

$$
x_{n}^{j}=\frac{(j+1 / 2) \pi}{n}-\frac{(j+1 / 2) \pi}{n} \frac{2}{5 n^{2} \pi}+\frac{2+\sin \left(\frac{j+1 / 2}{n}\right) \pi}{2 n^{2}}-\frac{6 / 5}{2 n^{2}}+o\left(\frac{1}{n^{2}}\right)
$$

then we can calculate that

$$
F_{0}(x)= \begin{cases}-\frac{4 x}{5 \pi}+\sin x+2 & \text { for } x<\frac{\pi}{2} \\ -\frac{4 x}{5 \pi}+\sin x+\frac{4}{5} & \text { for } x>\frac{\pi}{2}\end{cases}
$$


According to Theorem 1,

$$
\begin{aligned}
& h=\frac{F_{0}(0)}{2}=1, \\
& \mu_{0}=-F_{0}(\pi)+F_{0}(0)-F_{0}\left(\frac{\pi}{2}-0\right)+F_{0}\left(\frac{\pi}{2}+0\right)=\frac{4}{5}, \\
& q(x)+M(x, x)=F_{0}^{\prime}(x)+\frac{\mu_{0}}{\pi}=\cos x, \\
& \alpha=\sqrt{\frac{F_{0}(0)-2 F_{0}\left(\frac{\pi}{2}-0\right)}{F_{0}(0)-2 F_{0}\left(\frac{\pi}{2}+0\right)}}=2, \\
& H=\frac{\alpha^{+}\left(\mu_{0}-2 h\right)+2 \alpha^{-}\left(I_{1}(\pi / 2)-F_{0}(0)\right)}{\left(\alpha^{+}\right)^{2}-2 \alpha^{-}}=0 .
\end{aligned}
$$

\section{Conclusion}

In this paper we have investigated the discontinuous inverse nodal problem for Volterra type integro-differential operator. We showed that if one of $q(x)$ or $M(x, x)$ is given, then the other one can be determined uniquely by using only the given nodal points.

\section{Funding}

Not applicable.

\section{Abbreviations}

Not applicable.

\section{Availability of data and materials}

Not applicable.

\section{Ethics approval and consent to participate}

Not applicable.

\section{Competing interests}

The author declares that he has no competing interests.

\section{Authors' contributions}

The author read and approved the final manuscript.

\section{Publisher's Note}

Springer Nature remains neutral with regard to jurisdictional claims in published maps and institutional affiliations.

Received: 18 December 2017 Accepted: 26 March 2018 Published online: 03 April 2018

\section{References}

1. McLaughlin, J.R.: Inverse spectral theory using nodal points as data—a uniqueness result. J. Differ. Equ. 73, 354-362 (1988)

2. Hald, O.H., McLaughlin, J.R.: Solutions of inverse nodal problems. Inverse Probl. 5, 307-347 (1989)

3. Yang, X.-F.: A solution of the nodal problem. Inverse Probl. 13, 203-213 (1997)

4. Browne, P.J., Sleeman, B.D.: Inverse nodal problem for Sturm-Liouville equation with eigenparameter depend boundary conditions. Inverse Probl. 12, 377-381 (1996)

5. Buterin, S.A., Shieh, C.T.: Inverse nodal problem for differential pencils. Appl. Math. Lett. 22, 1240-1247 (2009)

6. Buterin, S.A., Shieh, C.T.: Incomplete inverse spectral and nodal problems for differential pencil. Results Math. 62 , 167-179 (2012)

7. Cheng, Y.H., Law, C.-K., Tsay, J.: Remarks on a new inverse nodal problem. J. Math. Anal. Appl. 248, 145-155 (2000)

8. Guo, Y., Wei, G.: Inverse problems: dense nodal subset on an interior subinterval. J. Differ. Equ. 255, 2002-2017 (2013)

9. Law, C.K., Shen, C.L., Yang, C.F.: The inverse nodal problem on the smoothness of the potential function. Inverse Probl. 15(1), 253-263 (1999). Erratum: Inverse Probl. 17, 361-363 (2001)

10. Ozkan, A.S., Keskin, B.: Inverse nodal problems for Sturm-Liouville equation with eigenparameter dependent boundary and jump conditions. Inverse Probl. Sci. Eng. 23(8), 1306-1312 (2015)

11. Shieh, C.-T., Yurko, V.A.: Inverse nodal and inverse spectral problems for discontinuous boundary value problems. J. Math. Anal. Appl. 347, 266-272 (2008)

12. Yang, X.-F.: A new inverse nodal problem. J. Differ. Equ. 169, 633-653 (2001) 
13. Yang, C.-F., Yang, X.-P.: Inverse nodal problems for the Sturm-Liouville equation with polynomially dependent on the eigenparameter. Inverse Probl. Sci. Eng. 19(7), 951-961 (2011)

14. Yang, C.-F.: Inverse nodal problems of discontinuous Sturm-Liouville operator. J. Differ. Equ. 254, 1992-2014 (2013)

15. Yang, C.-F., Pivovarchik, V.N.: Inverse nodal problem for Dirac system with spectral parameter in boundary conditions. Complex Anal. Oper. Theory 7, 1211-1230 (2013)

16. Buterin, S.A: On the reconstruction of a convolution perturbation of the Sturm-Liouville operator from the spectrum. Differ. Equ. 46, 150-154 (2010)

17. Buterin, S.A.: On an inverse spectral problem for first-order integro-differential operators with discontinuities. Appl. Math. Lett. 78, 65-71 (2018)

18. Freiling, G., Yurko, V.A.: Inverse Sturm-Liouville Problems and Their Applications. Nova Science, New York (2001)

19. Kuryshova, Y.V.: Inverse spectral problem for integro-differential operators. Math. Notes 81(6), 767-777 (2007)

20. $\mathrm{Wu}, \mathrm{B} ., \mathrm{Yu}, \mathrm{J}$.: Uniqueness of an inverse problem for an integro-differential equation related to the Basset problem. Bound. Value Probl. 2014, Article ID 229 (2014)

21. Yurko, V.A.: An inverse problem for integro-differential operators. Math. Notes 50(5-6), 1188-1197 (1991)

22. Yurko, V.A.: Inverse problems for second order integro-differential operators with discontinuities. Appl. Math. Lett. 74, 1-6 (2017)

23. Kuryshova, Y.V., Shieh, C.T.: An inverse nodal problem for integro-differential operators. J. Inverse III-Posed Probl. 18, 357-369 (2010)

24. Keskin, B., Ozkan, A.S.: Inverse nodal problems for Dirac-type integro-differential operators. J. Differ. Equ. 263, 8838-8847 (2017)

25. Buterin, S.A.: The inverse problem of recovering the Volterra convolution operator from the incomplete spectrum of its rank-one perturbation. Inverse Probl. 22, 2223-2236 (2006)

26. Buterin, S.A.: On an inverse spectral problem for a convolution integro-differential operator. Results Math. 50, 173-181 (2007)

\section{Submit your manuscript to a SpringerOpen ${ }^{\circ}$ journal and benefit from:}

- Convenient online submission

- Rigorous peer review

- Open access: articles freely available online

- High visibility within the field

- Retaining the copyright to your article

Submit your next manuscript at $\gg$ springeropen.com 